УДК 514.18

\title{
Я.А. Кокарева
}

Донской государственный технический университет,

Ростов-на-Дону, Россия

\section{АНАЛИТИЧЕСКАЯ МОДЕЛЬ ПОВЕРХНОСТЕЙ НА ОСНОВЕ КООРДИНАЦИИ ПРОСТРАНСТВА ВИНТОВЫМИ И ЭЛЛИПТИЧЕСКИМИ ЛИНИЯМИ}

\begin{abstract}
Рассмотрен алгоритм синтеза поверхностей путем погружения произвольной линии в конгруэнцию на основе конструктивно-параметрического метода формообразования. Получены параметрические уравнения координации пространства винтовыми и эллиптическими линиями, на основании которых определены $u$-, $v$ - и w-конгруэнции. Визуализированы поверхности полученных конгруэнций. Определена общая структура поверхностей.

Ключевые слова: конструктивно-параметрический метод, формообразование, конгруэнция линий, координация пространства, криволинейные координаты.
\end{abstract}

\section{la.A. Kokareva}

Don State Technical University, Rostov-on-Don, Russian Federation

\section{ANALYTICAL MODEL OF SURFACES BASED ON THE SPACE'S COORDINATION BY HELIX AND ELLIPSES}

In the article the algorithm of surface's construction on the basis of a construct-parametrical method of a shaping by immersion of any line in congruence is considered. The parametrical equations of coordination of space by helix and ellipses, on the basis of which $u$-, $v$ - and $w$-congruences are defined, are received. The surfaces of the resulting congruences are visualized. The general structure of surfaces is determined.

Keywords: construct-parametrical method, shaping, congruence of lines, space's coordination, curvilinear coordinates.

Формообразование поверхностей является неотъемлемой частью проектирования. С развитием технологий, методов расчетов, появлением новых материалов, позволяющих реализовать все более футуристические проекты, развиваются и методы формообразования поверхностей. 
Методы формообразования исторически развивались в соответствии с доступными способами визуализации и расчета конструкций: синтетический [1-3], конструктивно-синтетический [4-6], кинематический [7-9], метод преобразований [10, 11], метод криволинейного проецирования [12-14], параметрический [15], методы представления кусочно-аналитическими функциями $[8,16,17]$.

Наличие аналитической модели при проектировании сложных криволинейных форм упрощает расчеты конструкции. Однако часто по аналитическому представлению практически невозможно представить будущую форму поверхности. Одним из методов формообразования, который позволяет включить в уравнения поверхностей конструктивные элементы с задаваемыми параметрами, является конструктивно-параметрический метод, разработанный И.А. Скиданом и его учениками [18-22].

Цель данной статьи - синтез параметрических уравнений и визуализация поверхностей $u^{-}, v$ - и $w$-конгруэнций конструктивнопараметрическим методом на основе конструктивной схемы координации пространства винтовыми и эллиптическими линиями.

Суть конструктивно-параметрического метода сводится к тому, что используется конструктивная модель будущей поверхности в качестве переходной от задуманной к аналитической. При этом пространство параметризируется в соответствии с этой моделью, т.е. создается конструктивная схема координации пространства заданными линиями в качестве координатных. При этом, с одной стороны, пространство задается криволинейными координатами, а с другой - эти же координатные линии образуют три взаимосвязанных конгруэнции линий. Для образования поверхности используется погружение линии в одну из конгруэнций.

Приведем алгоритм синтеза параметрических уравнений поверхности, полученной путем погружения линии в конгруэнцию:

1. Создание конструктивной схемы координации пространства линиями.

2. Составление параметрических уравнений конгруэнции-координации в виде

$$
x=f_{1}(u, v, w) ; y=f_{2}(u, v, w) ; z=f_{3}(u, v, w) .
$$

С помощью уравнений (1) также задаются три конгруэнции $u$-, v- и $w$-линий. 
3. Выражение криволинейных координат через прямоугольные декартовы:

$$
u=\varphi_{1}(x, y, z) ; v=\varphi_{2}(x, y, z) ; w=\varphi_{3}(x, y, z) .
$$

4. Задание параметрических уравнений погружаемой в конгруэнцию линии:

$$
X=F_{1}(t) ; \quad Y=F_{2}(t) ; Z=F_{3}(t) .
$$

5. Подстановка уравнений (3) в уравнения (2) вместо $x, y, z$ соответственно:

$$
u=\varphi_{1}(X(t), Y(t), Z(t)) ; v=\varphi_{2}(X(t), Y(t), Z(t)) ; w=\varphi_{3}(X(t), Y(t), Z(t)) .
$$

6. В зависимости от выбранной конгруэнции линий $(u, v$ или $w)$ в уравнения (1) подставляются два уравнения (4), за исключением того, название которого входит в выбранную конгруэнцию. Например, для синтеза уравнений поверхностей конгруэнции $w$-линий в уравнения (1) подставляются выражения для $u$ и $v$ из уравнений (4):

$$
x=f_{1}(u(t), v(t), w) ; y=f_{2}(u(t), v(t), w) ; z=f_{3}(u(t), v(t), w) .
$$

Рассмотрим конструктивную схему координации пространства винтовыми и эллиптическими линиями (рис. 1):

- $u$ - параметр положения точки на заданной винтовой цилиндрической линии, являющейся местом центров сечений-эллипсов;

- $v$ - параметр главной оси эллипса;

- $w$ - параметр положения точки $M$ на эллипсе.

Фактически конструктивная схема представляет собой множество соосных винтовых поверхностей с подобными сечениями-эллипсами в плоскостях, перпендикулярных плоскости $X O Y$.

Согласно вышеприведенному алгоритму составим параметрические уравнения конгруэнции-координации.

В плоскости сечения $u=u_{0}$ уравнения плоского поля концентрических подобных эллипсов $(s-$ коэффициент соотношения полуосей эллипса) с учетом параметра-радиуса $a$ и параметра-шага $b$ винтовой линии будут иметь вид

$$
x^{\prime}=v \cos w+a ; y^{\prime}=s v \sin w+b u .
$$




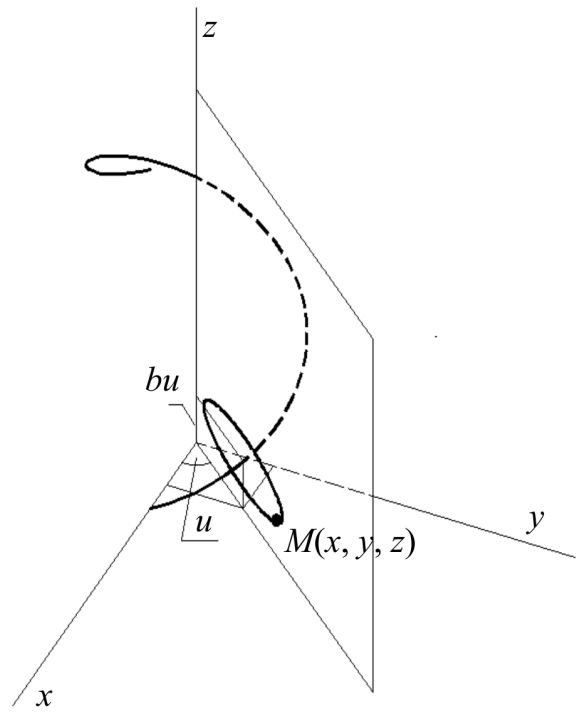

Рис. 1. Конструктивная схема координации пространства винтовыми и эллиптическими линиями

С учетом поворота плоскости найдем уравнения конгруэнциикоординации:

$$
\begin{aligned}
x=x^{\prime} \cos u & =(v \cos w+a) \cos u, \\
y=x^{\prime} \sin u & =(v \cos w+a) \sin u, \\
z=y^{\prime} & =s v \sin w+b u .
\end{aligned}
$$

Область определения функций (7) определяем, рассчитав якобиан и приравняв его к нулю:

$$
J=-v s(a+v \cos w)=0 .
$$

Анализируя выражение (8), можно определить, что при $v=0$ мы получаем уравнение винтовой цилиндрической линии, на которой располагаются центры эллипсов, а выражение в скобках определяет плоское поле эллипсов.

Координатные поверхности заданной криволинейной системы координат:

- $u=$ const - плоское поле концентрически расположенных эллипсов;

- $v$ = const - винтовая поверхность с сечением-эллипсом, перпендикулярным плоскости ХOY;

- $w=$ const - винтовая линейчатая поверхность. 
Координатные линии:

- $u$-линии - винтовые цилиндрические линии;

- v-линии - прямые линии;

- $w$-линии - эллипсы.

Далее при выполнении пунктов 3-6 приведенного алгоритма были получены изображения поверхностей $u^{-}, v$ - и $w$-конгруэнций (рис. 2,3 ).

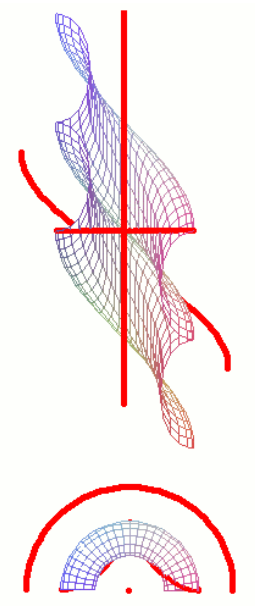

$a$

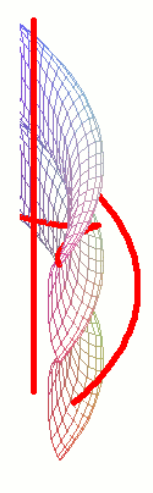

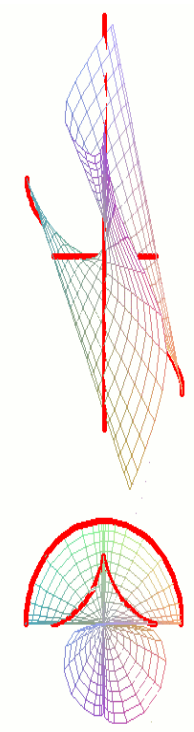

$\sigma$

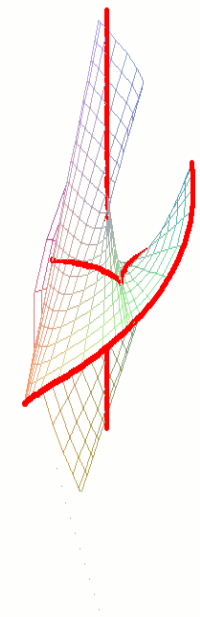

Рис. 2. Пример поверхности: $a-u$-конгруэнции; $\sigma-v$-конгруэнции
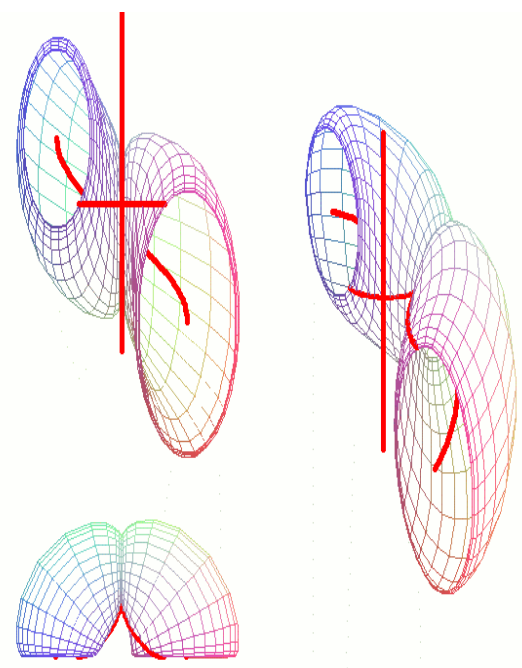

Рис. 3. Пример поверхности $w$-конгруэнции 
В конгруэнции (7) погружена астроида:

$$
X=\cos ^{3} t ; \quad Y=\sin ^{3} t ; \quad Z=2 .
$$

Постоянные параметры конгруэнции: $a=1,5, b=1, s=2$. Сами уравнения выражений (2) и поверхностей (5) не приведены из-за громоздкости полученного результата.

Рассмотрим структуру полученных поверхностей.

1. Поверхности $u$-конгруэнции являются винтовыми поверхностями, образующие которых пересекают погружаемую линию.

1. Поверхности $v$-конгруэнции являются линейчатыми поверхностями с тремя направляющими: осью $O Z$, погружаемой линией и винтовой цилиндрической линией с параметрами $a$ и $b$.

1. Поверхности $w$-конгруэнции являются каналовыми поверхностями с эллиптическими сечениями, перпендикулярными плоскости $X O Y$, центры которых образуют винтовую линию с параметрами $a$ и $b$. Поверхность содержит погружаемую линию.

При приближении к значениям $u=\pi n(n=0,1,2 \ldots)$ поверхности не определены.

Полученные типы поверхностей: винтовая, каналовая и линейчатая с тремя направляющими - могут быть использованы в технических проектах и дизайнерских решениях.

\section{Список литературы}

1. Reye T. Geometrie der Lage. - Leipzig, 1910. - V ol. 1-3.

2. Salmon G. A Treatise on the analytic geometry of three dimensions. - New Y ork: Chelsa Publishing Company, 1965 (reprint). - $486 \mathrm{p}$.

3. Sanger R.G. Synthetic projective geometry. - New Y ork; L ondon: McCraw - Hill Book Company, 1939. - 175 p.

4. Подгорный А.Л. Конструирование поверхностей оболочек по заданным условиям на основе выделения их из конгруэнций прямых // Прикладная геометрия и инженерная графика. - 1969. - Вып. VIII. - С. 17-28.

5. Підгорний О.Л., Несвідомін В.М. Створення комп'ютерних моделей нелінійчатої поверхні 3-го порядку методами синтетичної геометрії // Прикладная геометрия и инженерная графика. - 2008. Вип. 79. - С. 9-15.

6. Михайленко В.Е., Обухова В.С., Подгорный А.Л. Формообразование оболочек в архитектуре. - Киев: Будівельник, 1972. - 208 с. 
7. Короткий В.А., Усманова Е.А., Хмарова Л.И. Компьютерное моделирование кинематических поверхностей // Геометрия и графика. 2016. - T. 3, № 4. - C. 19-26. DOI: 10.12737/17347

8. Голованов Н.Н. Геометрическое моделирование. - М.: Изд-во физ.-матем. лит., 2012. - 472 с.

9. Кривошапко С.Н., Иванов В.Н. Энциклопедия аналитических поверхностей. - М.: Либроком, 2010. - 560 с.

10. Иванов Г.С. Конструирование технических поверхностей (математическое моделирование на основе нелинейных преобразований). - М.: Машиностроение, 1987. - 192 с.

11. Надолинный В.А. Основы теории проективных рациональных поверхностей: дис. ... д-ра техн. наук: 05.01.01. - М., 1989. - 202 с.

12. Обухова В.С. Двуосевое проектирование кривых линий // Прикладная геометрия и инженерная графика. - 1965. - Вып. І. - С. 39-47.

13. Обухова В.С. Обобщение нелинейных систем проекций и одноосевые системы // Прикладная геометрия и инженерная графика. 1970. - Вып. 10. - С. 17-27.

14. Сименко О.В. Проекціювання променями конгруенції циліндричних гвинтових ліній сталого кроку // Праці Таврійського державного агротехнічного університету. - 2004. - Т. 23, вип. 4. - С. 86-91.

15. Сальков Н.А. Параметрическая геометрия в геометрическом моделировании // Геометрия и графика. - 2014. - Т. 2, № 3. - С. 7-13. DOI: $10.12737 / 6519$

16. Замятин А.В., Кубарев А.Е., Замятина Е.А. Алгоритм аппроксимации поверхности сплайнами [Электронный ресурс] // Науковедение. - 2012. - № 3 (12). - URL: naukovedenie.ru/sbornik12/12-90.pdf (дата обращения: 12.01.2017).

17. Circular arc snakes and kinematic surface generation / M . Barton, L. Shi, M. Kilian, J. Wallner, H. Pottmann // Eurographics: Computer Graphics Forum. - Oxford: Blackwell Publishing Ltd., 2013. - Vol. 32, № 2. DOI: $10.1111 / \mathrm{cgf}$.12020

18. Сименко О.В. Аналітичні та комп'ютерно-графічні моделі нетрадиційних систем проекціювання та їхніх проекцію вальних поверхонь: дис. ... канд. техн. наук: 05.01.01. - Донецьк, 2006. - 216 с.

19. Скідан І.А., Кокарєва Я.А. Аналітичний опис еліптичної конгруенції прямих та іiї поверхонь // Праці Таврійського державного агротехнічного університету. - 2010. - Т. 48, вип. 4. - С. 36-43. 
20. Кокарєва Я.А. Аналітичні та комп'ютерні моделі поверхонь конгруенцій першого порядку прямих: дис. ... канд. техн. наук: 05.01.01. - Макіївка, 2011. - 203 с.

21. Кокарева Я.А. Параметрические уравнения конгруэнции прямых, заданной фокальными окружностями // Научное обозрение. 2014. - № 11-3. - С. 689-692.

22. Кокарева Я.А. Поверхности конгруэнции эквиаффинных образов окружности [Электронный ресурс] // Инженерный вестник Дона. 2016. - № 4. - URL: http: //www.ivdon.ru/ru/magazine/archive/n4y2016/3863 (дата обращения: 14.01.2017).

\section{References}

1. Reye T. Geometrie der Lage. L eipzig, 1910, vol. 1-3.

2. Salmon G. A Treatise on the A nalytic Geometry of Three Dimensions. New Y ork, Chelsa Publishing Company, 1965 (reprint), $486 \mathrm{p}$.

3. Sanger R.G. Synthetic projective geometry. New Y ork, London, McCraw - Hill Book Company, 1939, 175 p.

4. Podgornyi A.L. Konstruirovanie poverkhnostei obolochek po zadannym usloviiam na osnove vydeleniia ikh iz kongruentsii priamykh [The design of the surfaces of the membranes according to the preset conditions on the basis of their allocation of congruences direct]. Prikladnaia geometriia i inzhenernaia grafika. 1969, iss. VIII, pp. 17-28.

5. Pidgornii O.L., Nesvidomin V.M. Stvorennia komp'iuternikh modelei neliniichatoï poverkhni 3-go poriadku metodami sintetichnoï geometriï [The creation of computer models deliniate the surface of the 3-th order methods of synthetic geometry]. Prikladnaia geometriia $i$ inzhenernaia grafika. 2008, iss. 79, pp. 9-15.

6. Mikhailenko V.E., Obukhova V.S., Podgornyi A.L. Formoobrazovanie obolochek $v$ arkhitekture [The shaping of shells in architecture]. K iev, Budivel'nik, 1972, 208 p.

7. Korotkii V .A., U smanova E.A., K hmarova L.I. Komp'iuternoe modelirovanie kinematicheskikh poverkhnostei [Computer simulation of kinematic surfaces]. Geometriia i grafika. 2016, vol. 3, iss. 4, pp. 19-26, DOl: $10.12737 / 17347$.

8. Golovanov N.N. Geometricheskoe modelirovanie [Geometric simulation]. M oscow, Izd-vo fiziko-matematicheskoi literatury, 2012, 472 p. 
9. Krivoshapko S.N., Ivanov V.N. Entsiklopediia analiticheskikh poverkhnostei [Encyclopedia of the analytical surfaces]. M oscow, Librokom, 2010, 560 p.

10. Ivanov G.S. K onstruirovanie tekhnicheskikh poverkhnostei (matematicheskoe modelirovanie na osnove nelineinykh preobrazovanii) [Designing of technical surfaces (mathematical modelling on the basis of nonlinear transformations)]. M oscow, M ashinostroenie, 1987. $192 \mathrm{p}$

11. Nadolinnyi V.A. Osnovy teorii proektivnykh ratsional'nykh poverkhnostei [Fundamentals of the theory of projective rational surfaces]. Doctor's degree dissertation. M oscow, 1989, 202 p.

12. O bukhova V.S. Dvuosevoe proektirovanie krivykh linii [T wo axial projecting curves]. Prikladnaia geometriia $i$ inzhenernaia grafika. 1965, iss. I, pp. 39-47.

13. Obukhova V.S. Obobshchenie nelineinykh sistem proektsii i odnoosevye sistemy [Generalization of nonlinear systems of projections and the one-axis system]. Prikladnaia geometriia i inzhenernaia grafika. 1970, iss. 10, pp. 17-27.

14. Simenko O.V. Proektsiiuvannia promeniami kongruentsiï tsilindrichnikh gvintovikh linii stalogo kroku [The projection rays of congruence cylindrical helix with a constant pitch]. Pratsi Tavriis'kogo derzhavnogo agrotekhnichnogo universitetu. 2004, iss. 4, vol. 23, pp. 86-91.

15. Sal'kov N.A. Parametricheskaia geometriia $v$ geometricheskom modelirovanii [Parametric Geometry in Geometric M odeling]. Geometriia $i$ grafika. 2014, vol. 2, iss. 3, pp. 7-13, DOI: 10.12737/6519.

16. Zamiatin A.V., Kubarev A.E., Zamiatina E.A. Algoritm approksimatsii poverkhnosti splainami [A lgorithm of approximation of a surface splines]. Naukovedenie. 2012, no. 3, iss. 12, available at: naukovedenie.ru/ sbornik12/12-90.pdf

17. Barton M., Shi L., Kilian M., Wallner J., Pottmann H. Circular A rc Snakes and Kinematic Surface Generation. Computer Graphics Forum "Eurographics". Oxford, Blackwell Publishing Ltd, 2013, vol. 32, no. 2, DOI: $10.1111 /$ cgf.12020.

18. Simenko O.V. A nalitichni ta komp'iuterno-grafichni modeli netraditsiinikh sistem proektsiiuvannia ta ïknikh proektsiiu val'nikh poverkhon' [A nalytical and computer-graphics model of unconventional systems of the projection and the projection tive surfaces]. Ph. D. thesis. Donetsk, 2006, $216 \mathrm{p}$. 
19. Skidan I.A., K okareva Ia.A. Analitichnii opis eliptichnoï kongruentsiï priamikh ta ï̈ poverkhon' [A nalytical description of congruence elliptical straight and its surfaces]. Pratsi Tavriis'kogo derzhavnogo agrotekhnichnogo universitetu. 2010, iss. 4, vol. 48, pp. 36-43.

20. Kokareva Ia.A. A nalitichni ta komp'iuterni modeli poverkhon' kongruentsii pershogo poriadku priamikh [A nalytical and computer models of the surfaces congruence first order direct]. Ph. D. thesis. Makeevka, 2011, $203 p$.

21. Kokareva Ia.A. Parametricheskie uravneniia kongruentsii priamykh, zadannoi fokal'nymi okruzhnostiami [Parametric equations of the congruence of straight lines preset by focal circumferences]. Nauchnoe obozrenie. 2014, no. 11-3, pp. 689-692.

22. Kokareva Ia.A. Poverkhnosti kongruentsii ekviaffinnykh obrazov okruzhnosti [The surface of congruency equiaffine images of the circle]. Inzhenernyi vestnik Dona. 2016, no. 4, available at: http: //www.ivdon.ru/ ru/magazine/archive/n4y2016/3863.

Получено 26.02.2017

\section{Об авторе}

Кокарева Яна Андреевна (Ростов-на-Дону, Россия) - кандидат технических наук, доцент кафедры «Информационные системы в строительстве», Донской государственный технический университет (344000, Ростов-на-Дону, пл. Гагарина, 1, e-mail: kokareva.ya.a@ gmail.com).

\section{About the author}

Iana A. Kokareva (Rostov-on-Don, Russian Federation) - Ph.D. in Technical Sciences, A ssociate Professor, Department of Information System in the Civil Engineering, Don State Technical University (1, Gagarin Square, Rostov-on-Don, 344000, Russian Federation, e-mail: kokareva.ya.a@ gmail.com). 